\title{
Habitat Demonstration Unit Pressurized Excursion Module Systems Integration Strategy
}

\author{
T.R.Gill ${ }^{1}$, J. C. Merbitz ${ }^{2}$ \\ NASA Kennedy Space Center, KSC, FL 32899 \\ K. J. Kennedy ${ }^{3}$, L. Toups ${ }^{4}$, T. O. Tri ${ }^{5}$ \\ NASA Johnson Space Center, Houston, TX 77058 \\ A. S. Howe 6 \\ NASA Jet Propulsion Laboratory, Pasadena CA 91109
}

\begin{abstract}
I. Abstract
The Habitat Demonstration Unit (HDU) project team constructed an analog prototype lunar surface laboratory called the Pressurized Excursion Module (PEM). The prototype unit subsystems were integrated in a short amount of time, utilizing a rapid prototyping approach that brought together over 20 habitation-related technologies from a variety of NASA centers. This paper describes the system integration strategies and lessons learned, that allowed the PEM to be brought from paper design to working field prototype using a multi-center team.

The system integration process was based on a rapid prototyping approach. Tailored design review and test and integration processes facilitated that approach. The use of collaboration tools including electronic tools as well as documentation enabled a geographically distributed team take a paper concept to an operational prototype in approximately one year. One of the major tools used in the integration strategy was a coordinated effort to accurately model all the subsystems using computer aided design (CAD), so conflicts were identified before physical components came together.

A deliberate effort was made following the deployment of the HDU PEM for field operations to collect lessons learned to facilitate process improvement and inform the design of future flight or analog versions of habitat systems. Significant items within those lessons learned were limitations with the CAD integration approach and the impact of shell design on flexibility of placing systems within the HDU shell.
\end{abstract}

\section{Background}

A technique being utilized in NASA's exploration architecture analysis is rapid prototyping for analog testing in relevant environments such as desert locales as seen in Ref. 1. Running through potential "day in the life" scenarios at an outpost with prototype equipment allows designers insight into the utilization of the proposed systems and refines architecture and operations concepts. A series of Desert Research and Technology Studies (RaTS) have been held in locations such as Moses Lake, Washington, Black Point Lava Flow, and SP Crater, Arizona, where the most recent tests in September 2010 were performed simulating a 14-21 day lunar mission. A depiction of the lunar architecture $^{2}$ under evaluation in the Desert RaTS 2010 campaign is depicted in Figure 1.

1 Engineer, Lunar Systems Division, Mail Code LX-M,

2 Engineer, Lunar Systems Division, Mail Code LX-M,

3 Architect, Systems Architecture and Integration Office, Mail Code EA3

4 Engineer, Exploration Missions and Systems Office, Mail Code ZS

5 Engineer, Systems Architecture and Integration Office, Mail Code EA3

6 Architect, Mission Systems Concepts, Mail Code 312E 


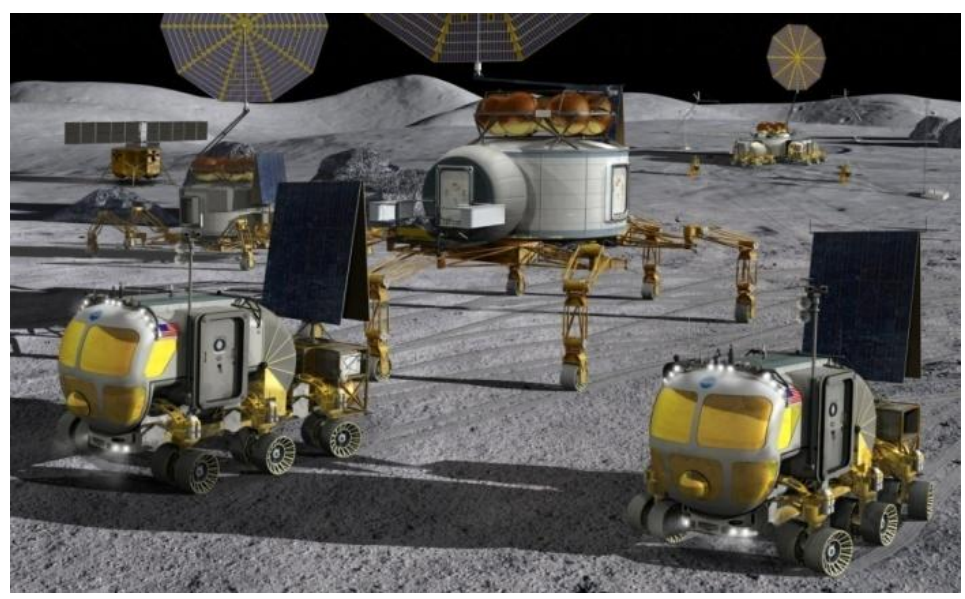

Figure 1 - Example of a lunar architecture analyzed through Desert RaTS campaigns.

The NASA hardware demonstrated by Desert RATS 2010 included: Space Exploration Vehicles - a pair of rovers that astronauts lived in for 14 days at a time; Habitat Demonstration Unit/Pressurized Excursion Module - a simulated habitat where the rovers docked to allow the crew room to perform experiments or deal with medical issues; Tri-ATHLETEs, or All-Terrain Hex-Legged Extra-Terrestrial Explorer - two heavy-lift rover platforms that allowed transportation of large items; a Portable Communication Terminal Concept (PCT) to facilitate communication with mobile assets; Centaur 2 - a four-wheeled transportation option for NASA Robonaut 2; Portable Utility Pallets (PUPs) for short - mobile charging stations for equipment; and a suite of new geology sample collection tools, including a self-contained GeoLab glove box for conducting in-field analysis of various collected rock samples.

The HDU PEM went from a Constellation Lunar architecture concept in June 2009 to an operational prototype in August 2010. When evaluating design considerations for the Habitat Demonstration Unit, the two primary areas of discussion are the integration strategy, which covers the processes and approach to enable rapid prototype development, and lessons learned, which can be applied to future prototypes and habitats.

\section{Integration Strategy}

The HDU project has required integrating a variety of contributions from NASA centers and has posed a challenge in integrating these disparate efforts into a cohesive architecture. The HDU began its work as an emulation of a Pressurized Excursion Module (PEM) for 2010 field testing. The PEM concept is a mobile lunar laboratory that works in concert with crews in SEV rovers for science sample analysis and maintenance tasks rather than a traditional habitat. Preliminary plans were to evolve the HDU to a Pressurized Core Module (PCM), which is more of a traditional habitat with crew accommodations, for 2011 and later field tests. However, the changing of priorities in NASA direction has influenced the future HDU configuration to a more flexible version of a habitat and laboratory. The HDU PEM deployment was not the same configuration as either the PCM or the PEM as conceived in Constellation lunar architecture plans. There were some developmental hardware and software items that were not available due to cost and technology availability and some items that were only functional simulations. Conversely, there were additional items available for the HDU that were opportunities for demonstration, which were originally unanticipated systems that were plugged into the HDU architecture because they had common goals and objectives. Results of the HDU field tests will influence future designs of exploration habitats.

Successfully deploying the HDU PEM, from paper concepts to actual demonstration hardware in just over one calendar year, required the HDU project team to utilize a rapid prototyping approach at the core of the integration strategy. The team focused energy on shell design initially as that design paved the way for the manufacturing and assembly operations. The design review and integration processes and collaborative tools utilized were also critical to the rapid prototyping approach. The unique aspect of this integration strategy was to utilize advanced systems engineering processes at a level tailored for a rapid prototyping environment. This novel approach had not previously been utilized at NASA on an operational habitat system. 


\section{A. Shell Design}

Early in the design process, it was recognized that the HDU PEM shell would be a very large structure with its $5 \mathrm{~m}$ diameter and $3.5 \mathrm{~m}$ height. Because of the size, the overall design and the transportation of the shell influenced one another. To ensure that the HDU shell would meet the project expectations and requirements, which directly affected how the shell would be shipped, a shell design and manufacturing requirements document was developed. At this point in the project, the team needed to determine the best method for shipping the HDU PEM. To do so, the project team performed a trade analysis to determine the best configuration for shipping the HDU PEM and the best means of transportation for the shipment. When the trade analysis was complete, the transportability trade assessed the following 3 options: (1) to transport the HDU fully assembled as a supersize load, (2) to transport the HDU in a single-split configuration as a standard load, and (3) to transport the HDU in a dual-split unit as a standard load.

A complete assessment of the three options concluded shipping an integrated HDU as a super-size load even though the super-size load is about 10 times more costly than a standard tractor-trailer load. The split configurations added costs for manufacturing a more complex split configuration shell; requiring in-field assembly; special transport support equipment and coverings, and additional ground support equipment. Finally, the increased amount of field assembly work required at the deployment would have added additional risk. Thus the benefits of transporting as a supersize load, out-weighed all the additional costs and operations if shipped as a standard load.

The decision to transport the HDU as one integrated super sized load required a transportation cradle to be designed to support the three purposes of (1) integration and testing at Johnson Space Center, (2) transportation to field locations, to other centers, or to outreach activities, and (3) to support field operations in the desert analog locations. In addition, for field operations the cradle acted as an analog substitute for the All-Terrain Hex Legged Extra-Terrestrial Explorer (ATHLETE) rover. Lastly, the cradle was fitted with heavy-duty jack stands for adjustment of the height and leveling to be able to configure for docking with the Space Exploration Rovers (SEVs) in the Desert RaTS and dry run trials. Figure 2 below displays the CAD and fabricated version of the HDU Cradle.

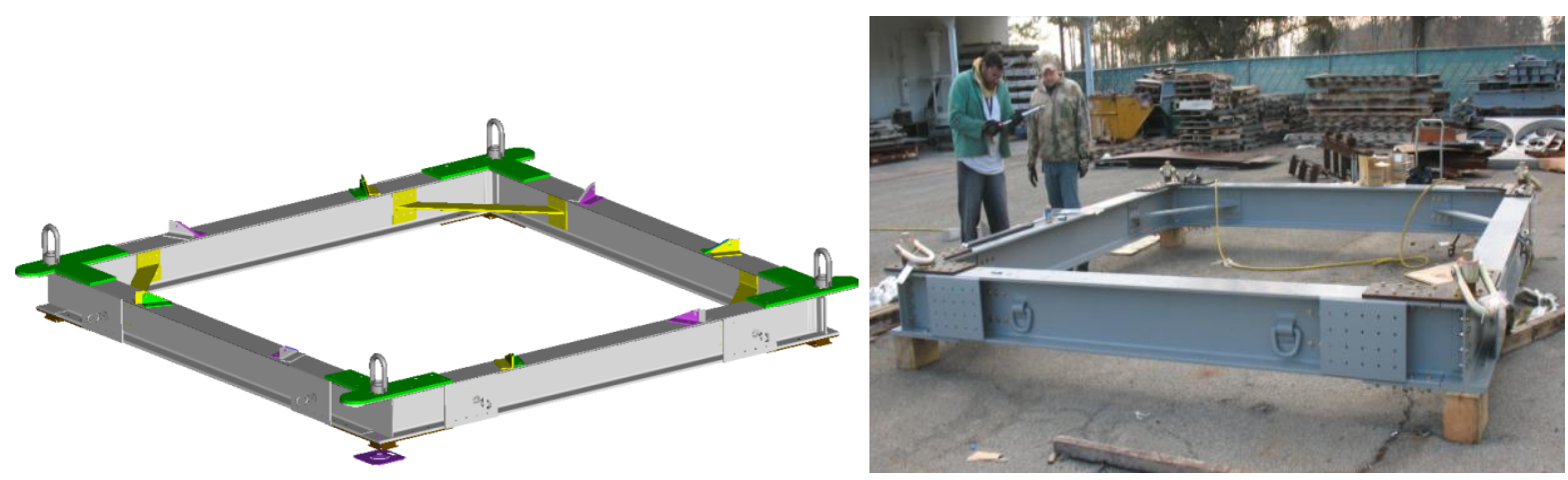

Figure 2 - HDU Cradle

Key factors in designing the shell of the HDU were cost, simplicity, and functionality. The team wanted a shell that would provide strength and stability but at the same time be made out of something light weight. Also, the shell needed to be constructed under a limited budget, and using a fiberglass composite shell enabled a cost effective manufacturing approach. With influence from the transportation assessment, the team decided on an "orange slice" design, which basically sectioned the shell of the HDU like orange slices so when it all came together, it created one cohesive shell. The HDU shell slices were created with a composite material that had steel ribs at either end to provide a bolting surface to attach each slice together, which ultimately created a double steel rib at each joint. Because each shell piece was created from one common mold, creating each piece of the shell was fairly simple, even when small modifications to the mold were required to compensate for a window or doors. Examples of the HDU rib and the mold for the shell slices are seen below in Figure 3. 

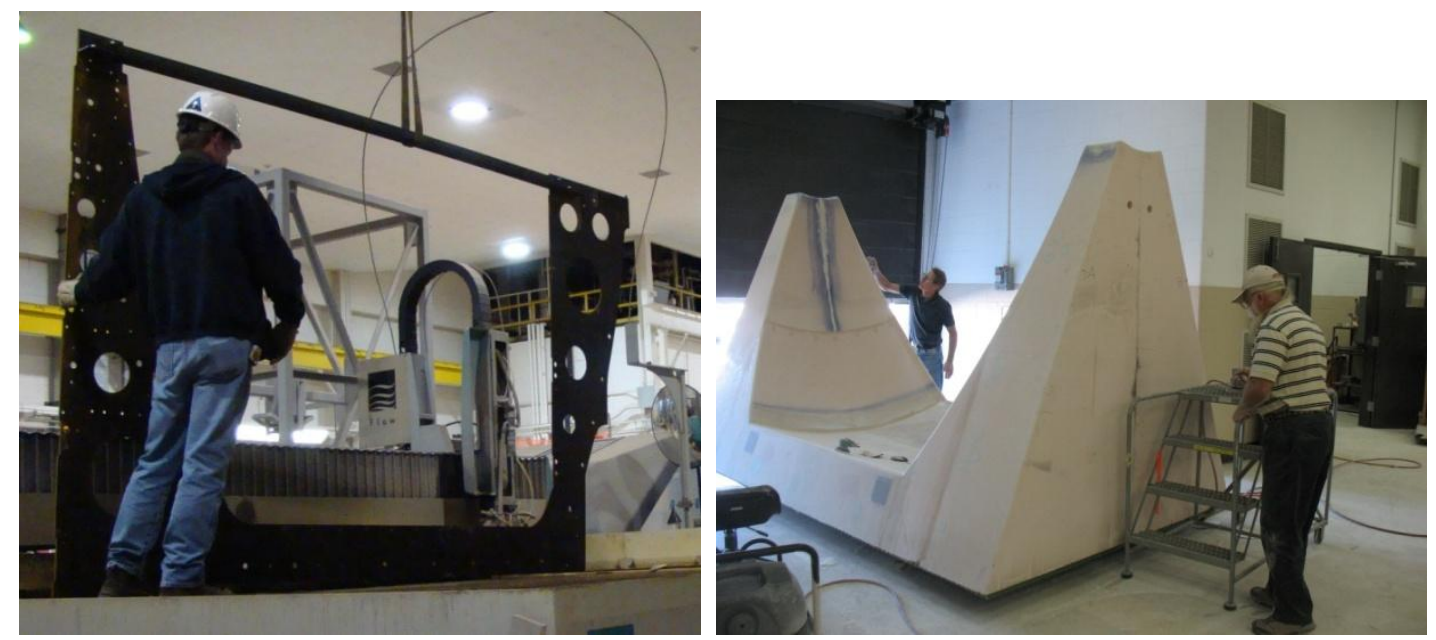

Figure 3 - An HDU Rib being removed from the water-jet machine (left) and the HDU shell slice mold during its fabrication (right)

When shell fabrication was complete at Langley Research Center in Virginia, the individual slices were shipped to JSC where they were integrated to create the HDU shell. Originally the concept was to potentially begin integration of subsystem components into the individual shell sections before all 8 shells were connected, but since fabrication of the shells went so smoothly, the shell was assembled and ready for integration in late February 2010 ahead of the planned beginning of subsystem integration in March 2010.

\section{B. Tailored Review Process}

A total of six NASA centers participated on the HDU-PEM deployment. When six different centers collaborate on a project, problems can occur due to poor communication between geographically distributed team members. The HDU Project Management conducted a series of reviews, in addition to weekly tag up meetings, to ensure the project ran with minimal complications. There were seven key reviews held during the campaign to deploy the HDU PEM between the summer of 2009 and the summer of 2010. These reviews are a tailored version of a typical NASA System Engineering design process.

The first review was the System Definition Review (SDR). The purpose of the SDR was to collaborate as a team to define the basic concepts for the systems that would constitute the HDU Pressurized Excursion Module. This included the systems, the workstations, and the technology demonstrations. This early definition meeting laid out the basic system concepts and set the stage for the teams to mature those concepts and begin envisioning integrating those systems together into an operational system.

The second review was the System Implementation Review (SIR). Here each subsystem identified the hardware being designed or selected and defined a preliminary plan for integration into or on the HDU-PEM. For example, some systems required minimal labor with no electrical power where others required a week of labor, electrical, mechanical, and crane support. Because each subsystem was unique, it was important for the team to come up with a preliminary integration plan and discuss it in an integrated team forum. In instances when two subsystems may interfere or perhaps have common work that could be combined, this review would bring to light opportunities to resolve problems or conversely to maximize efficiency.

The third review was the Computer Aided Design (CAD) Integration Review (CIR). Before this review, each system either developed a CAD model of their contribution or worked with the CAD integrator to develop models representative of their system. In some cases, these models were high fidelity models used to manufacture hardware. In other cases, they were simple shapes modeled to represent commercial-off-the-shelf hardware. This review was one of the most important reviews because the visual representation allowed the team to look at the fit, form, and function of the hardware in an integrated CAD model. So not only did the team discuss how the hardware systems would be populated within the HDU PEM but, by importing the model into a simulation tool, the team could manipulate and move elements to show their operations and use operability as a design consideration. In many cases, the overall CAD model pointed out some significant issues such as hardware interferences, cable routing problems, and human factors and sizing issues, which could be corrected before "cutting metal". Figure 4 
below shows representative samples from the integrated CAD model of the HDU PEM, while Figure 5 shows photos of the final results of the integration activities within the HDU shell.

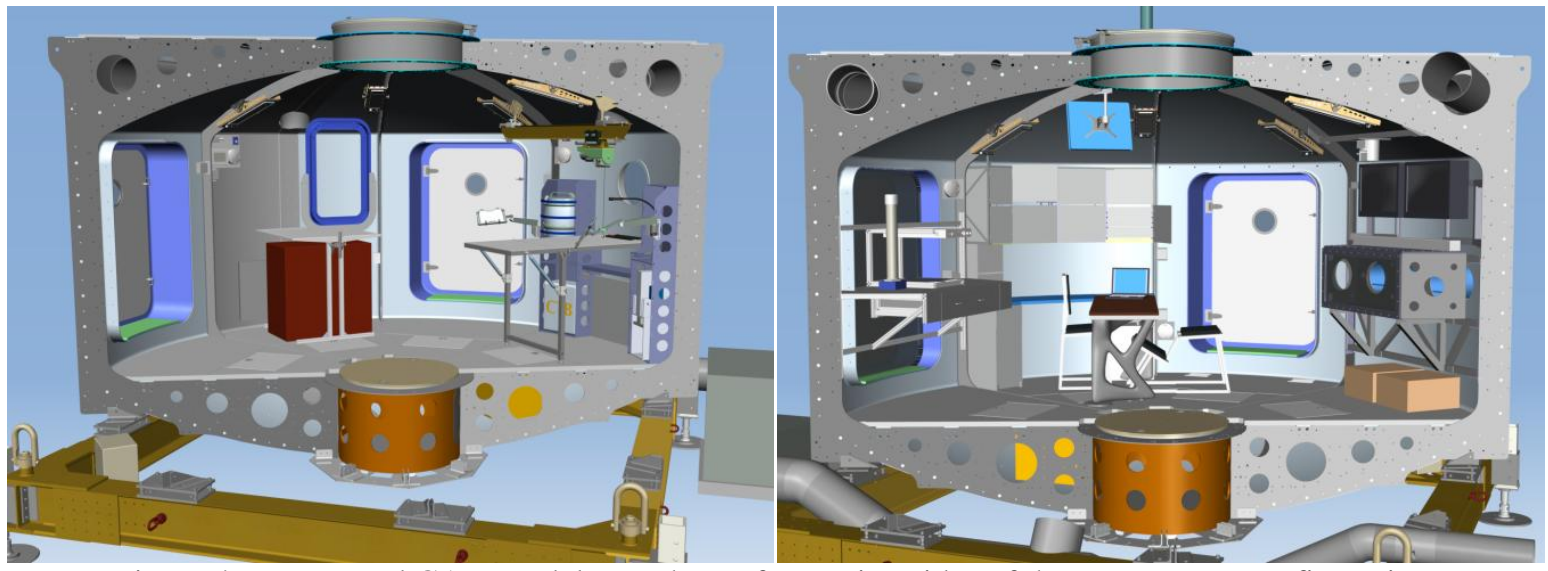

Figure 4 - Integrated CAD model snapshots of opposing sides of the HDU-PEM configuration
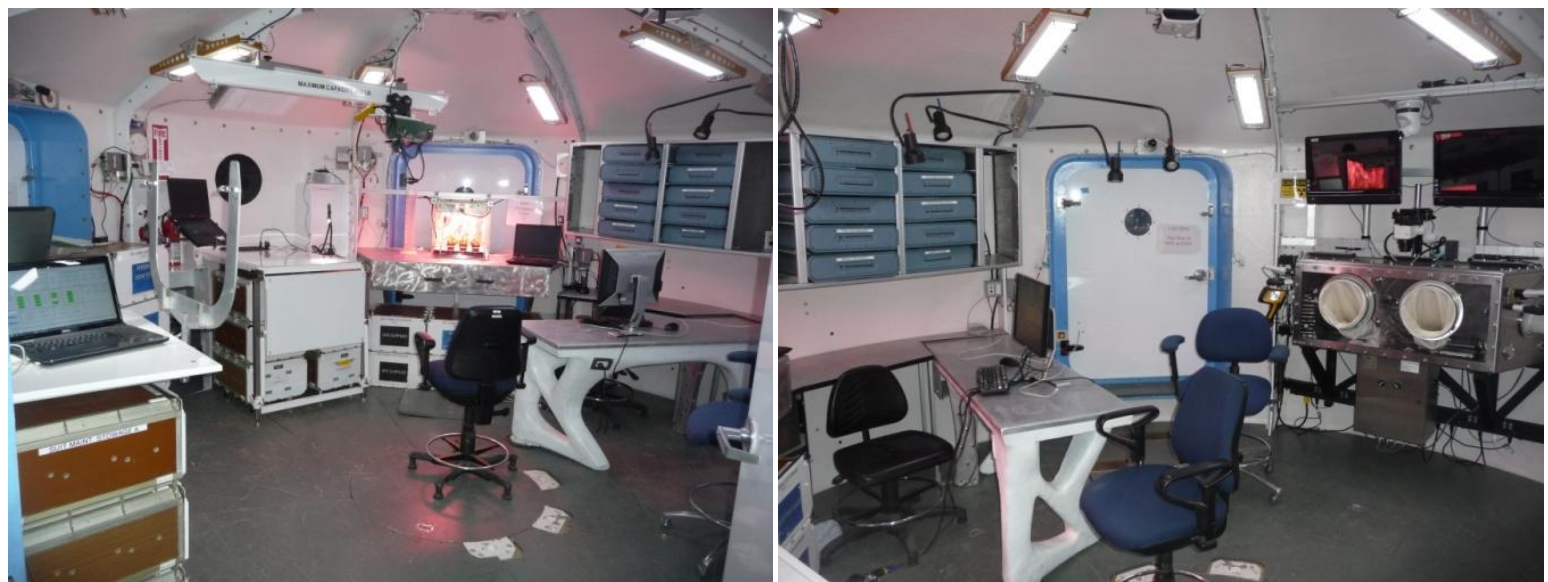

Figure 5 - Interior views of the HDU PEM as seen during the Desert RaTS 2010 campaign

Fourth in the series of reviews was the Preliminary Integration Review (PIR). This review is fairly close in function to a Preliminary Design Review (PDR). At this review, each subsystem began to finalize their system designs and delivered their contribution to the PEM Master Equipment Lists (MEL) and the integrated electrical schematic. Additionally, the system integration scheme was further matured within the integrated CAD model.

The fifth review, the final review regarding integration, was the Final Integration Review (FIR). This review is fairly close in function to a Critical Design Review (CDR) and included a final general system overview, hardware deliverables, and test objectives for each subsystem. It also included summaries of the electrical schematics, master equipment list, and CAD model snapshots for each system. Finally, a preliminary integration work plan for each system was defined to plan the integration tasks to occur in the next few months. .

The sixth review was the Test Configuration Review (TCR). The TCR defined the final configuration of the HDU PEM that would be tested during field test operations. This review gave a brief review of previously covered areas, identified any changes that occurred during the integration process, and provided a brief description of the materials to be used to develop training and operations material.

Last was the Test Readiness Review (TRR). There were actually two TRRs held. The first was the Integrated Systems Test TRR and the second was held for operational field testing. The Integrated Systems Testing was the first time when all the systems were integrated and considered operational together. It consisted of test sequences with multiple systems operating at the same time to evaluate potential interferences and to collect performance information. The Integrated Systems Test was intended to be performed indoors at JSC Building 220 in June 2010, but it actually occurred outside at the JSC Rock Yard in July 2010 because of schedule concerns. The second of the 
two reviews was the Field Testing TRR, which involved the operations to deploy and operate the HDU systems in an operational environment.

In each of these reviews, the team addressed actions from previous reviews, the risks, and forward work, and new actions were identified as the team progressed on the design and deployment of the HDU PEM.

\section{Tailored Test Operations}

Because of the complexity of deploying an operational prototype for the first time, a series of tailored test operations for the HDU-PEM were designed to prepare the team, the hardware and software, and procedures for the eventual Desert RaTS activities. With the large number of systems being integrated and tested for the HDU-PEM it was important to have a common document that had descriptions, testing procedures, and operational procedures for each system. In an attempt minimize documentation development, an HDU-PEM Operations Manual was created. The Operations Manual consisted of backgrounds on every subsystem, procedures required for each subsystem, and scripted scenarios required for field activities. This Operations Manual was also used for the training and certification of HDU PEM operators.

The first phase of the testing involved the Habitat Testbed (HaT) where key subsystems and simulation tools of the HDU PEM were laid out on a bench top test bed. This HaT capability allowed the team to perform tests on each PEM system prior to integration in the HDU shell. That allowed the HDU team to test and debug systems prior to integration in the HDU PEM to minimize troubleshooting on post installation test operations. These tests were done throughout the integration phase from March 2010 through July 2010 as hardware was delivered to be integrated into the HDU PEM. The next phase included the individual system tests that verified each independent system was performing as expected following integration activities, again between March and July 2010. The Integrated System Test, mentioned earlier, was a test to evaluate and measure performance of systems operating together for the first time. This test occurred in early July 2010. Additionally, some scripted test activities to support fieldtesting were practiced such as the activation and deactivation sequences, the emergency shutdown sequence, and the transitions between active and quiescent modes. The next phase between mid July and early August 2010 was a period of dry run tests performed at the Johnson Space Center Rock Yard where Lunar and Martian surface simulations exist. These Rock Yard tests allowed the team to exercise procedures not only for system testing but also for loading, transportation, unloading, and setup. These tests also engaged the Space Exploration Vehicle rovers, and all procedures run at the Desert RATS 2010 campaign were first executed at the Rock Yard. Finally, the culmination of all the test and integration activities was the utilization of the HDU PEM in Arizona to support the Desert RaTS 2010 field test operations from late August through mid-September of 2010. For additional detail on the field test operations, see Ref. 3. At each of these phases of testing, the same Operations Manual was utilized and updated, again, to minimize required documentation development. A high level overview showing the timeline of these activities can be seen in the HDU PEM project schedule summary chart seen in Figure 6. 


\section{HDU1 FY10 Project Schedule}

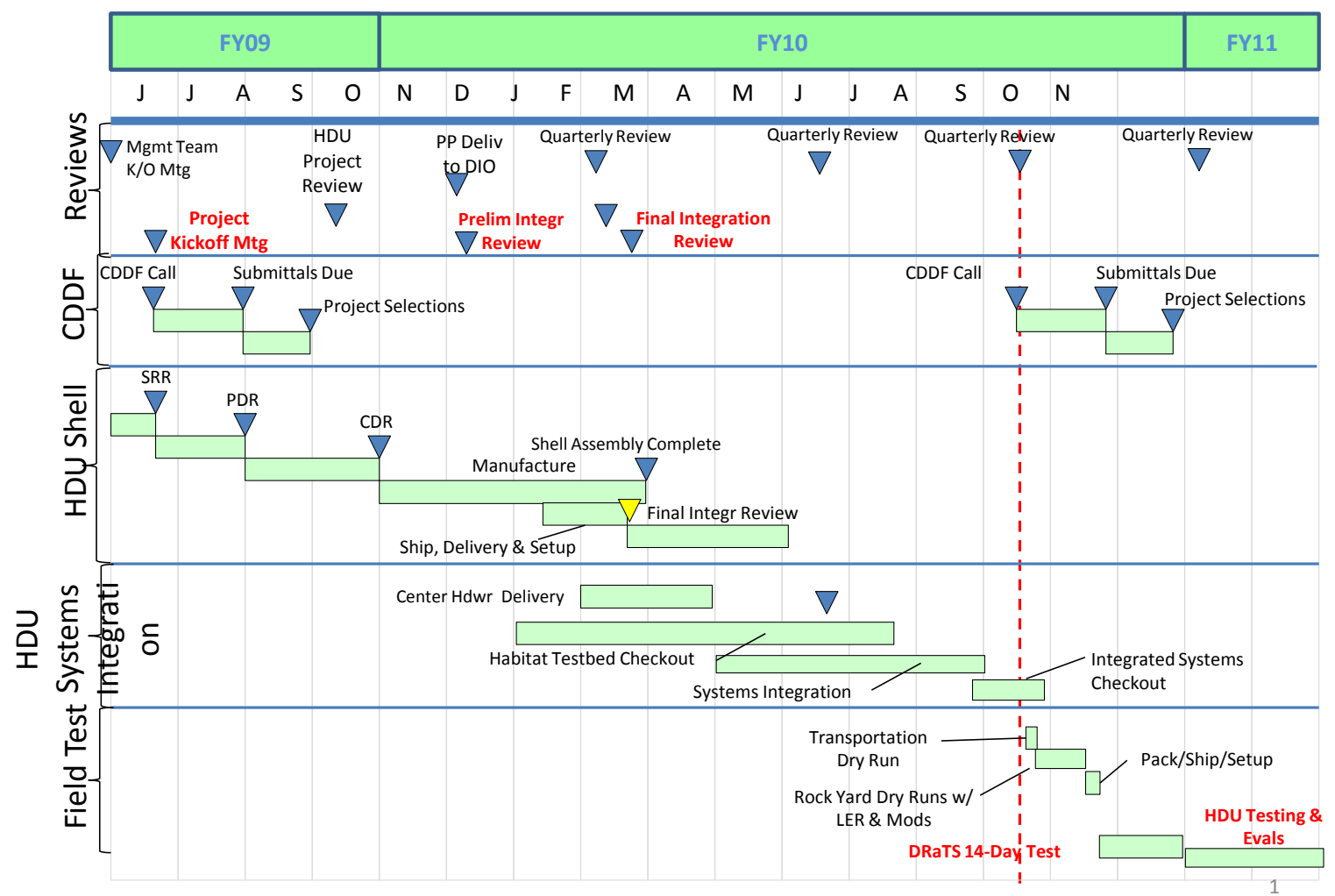

Figure 6 - Overview of HDU PEM project schedule

\section{Collaborative Tools}

During these reviews, the team utilized commercial meeting tools that allowed for file and information sharing to provide an accommodating means of communication. Before each meeting, each subsystem would upload their presentation files to a data repository using Windchill collaboration software. After acquiring access to this system, each HDU team member would have access to all meeting documents and presentations posted, which provided a valuable means of reference. The HDU project management team managed a wiki to act as a home page for meeting agendas and announcements and used it as portal by providing convenient links to the appropriate areas of the Windchill repository for schedule, review material, and other documentation. As many geographically distributed teams operate, team members would call into a common teleconference number and use desktop sharing software to conduct the meetings for online meeting collaboration. Conducting team meetings and reviews in this fashion are becoming commonplace for distributed teams, but having the tools to operate this way is still critical to being effective.

The HDU Project team utilized several key documents to record key information during the HDU integration and testing process. These documents were the Integration Plan, Work Plans, and the aforementioned Operations Manual. The Integration Plan was a summary document with excerpts pulled from the design reviews where the system overviews and internal and external interfaces were documented for current and future versions of the HDU. For example, the various types of power to be provided for HDU systems and the bolt patterns of the ribs for secondary structure attach points were all defined for the benefit of other systems. The Work Plans for each system were general outlines of the physical integration work to be performed for each system including shell protrusions and special tooling, materials, or personnel requirements. All of these plans and manuals were products of the reviews held during the project.

The General Maintenance Work Station (GMWS) was one system that required several configuration steps to become operational for test activities. After writing the integration plan and work plan and performing the integration work, the Operations Manual became an enhanced version of the other documents pointing out the 
necessary steps for use, as well as safety and potential risks. As seen in many engineering applications, actual system operations reflect more than what is learned through simulated operations using software tools. Lessons learned are reflected in the procedures so that when the time comes to use a system during planned field operations, the activities can be performed nominally. Again on the General Maintenance Work Station, the Operations Manual provided descriptions on deploying and stowing the work table, operating the computer mounts, and safely operating the hoist for lifting large items onto the GMWS table surface for repair or maintenance. Figure 7 shows the CAD and an image of the GMWS and points out the functions the station provided as seen in the Operations Manual.

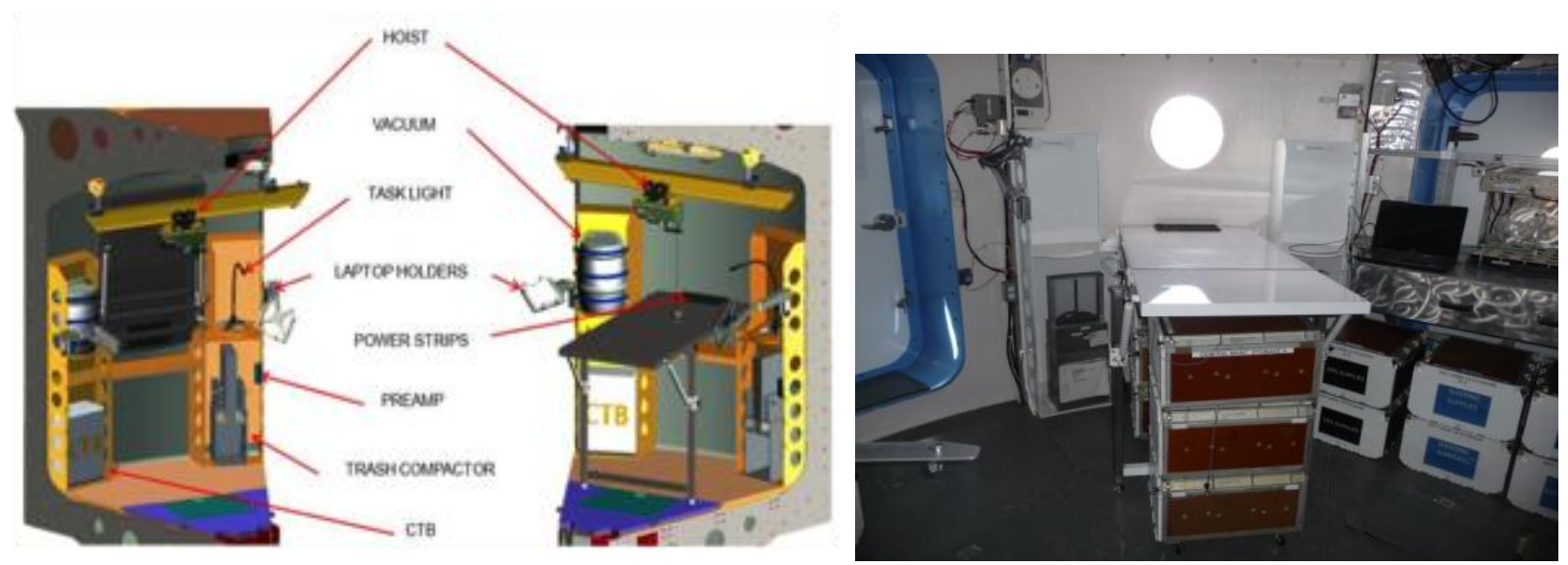

Figure 7 - General Maintenance Work Station (GMWS)

Ultimately, the final documentation product, the Operations Manual, became a valuable training tool and reference manual for the subsystem teams during dry-run testing at the JSC Rock Yard and for the crew participating in Desert RaTS activities at Black Point Lava Flow, just outside of Flagstaff, Arizona.

\section{Lessons Learned}

Many projects in the aerospace industry take months, years, and sometimes even decades from concept to actuality. The reason for this is due to all the factors that play a role into making a project successful. Budget, schedule delays, missed milestones, problems with design, problems with fabrication, and problems with testing are some common areas where issues occur during a project life. As with most aerospace projects, it is a rare case that one rendition of a spacecraft, for example, would be built and tested. With each additional year a program is extended, modifications to previous or completely new pieces of hardware are built for use during the next generation. To ensure that mistakes aren't repeated and that problems are identified from all previous years of a project, teams must take into account the lessons learned and apply them.

The purpose of lessons learned is to compile insights and observations a team may have documented during a project so they can be applied to future projects. At the conclusion of the FY2010 effort with HDU including design, integration, testing, and the field campaign, the multi-center HDU team collected its own lessons learned and synthesized them as part of the overall Desert RaTS team lessons learned capture process.

There are three significant areas of impact revealed by the HDU PEM lessons learned solicitation: (1) computer aided design, (2) integration, and testing and (3) thermal system selection. During the HDU project, the project team utilized simulation tools to assist in the design layout of the HDU subsystems. Computer Aided Design (CAD) representations of the various HDU systems were used during the Integration Review process to determine where systems would be located internal and external to the HDU shell prior to beginning the touch-labor integration effort. The CAD model was used for layout of subsystems and evaluation of sizing, cable lengths, and operational issues prior to physical integration. After coordinating with each system for initial recommendations on placing systems most effectively and conveniently, two integration reviews were held to include the entire team where the virtual model of the HDU was utilized. Additionally, once the integrated model of the HDU-PEM was available, it was utilized in a simulation tool where operations including unloading and setup of systems in the field and docking with the SEV Rovers were evaluated. The direction of docking of the SEV Rovers with respect to the HDU-PEM to avoid several potential mechanical interferences is an example of resulted from the simulation. Continuing into the 
physical integration phase, the model was continually updated to reflect the changes performed in the field to provide a starting point for future planning activities.

The team made efforts to identify where all the individual subsystems would be installed during the CAD design, but there were still lessons to be learned from the physical integration that informed the overall system design. The CAD model developed for HDU PEM was of medium fidelity to plan integration activities and did not include the cabling, for example, to reduce the level of effort in a rapid prototyping effort, allowing for some field engineering decisions by an experienced integration team. Thus the HDU team used CAD modeling at a level of effort deemed cost effective for a rapid prototyping effort rather than develop a more detailed model that might be utilized for a Building Information Modeling (BIM) effort. An example of the lessons learned in this area of CAD integration was with the internal lighting system for the HDU. The internal lighting system of the HDU consisted of 8 solid-state lighting modules (SSLMs), which were to be powered by a power distribution unit (PDU), and controlled through a controller. In order to power and control the lights, cabling had to be run throughout the HDU to each individual light. By using CAD, the team was able to not only identify the best locations for the light installation, but also identify cable lengths, and where the power and control boxes could be installed without interfering with any other subsystems. But when the team arrived to begin integrating the lighting subsystem, the HDU was not in the configuration the team shown in the CAD model. For example, the terminal box and the controller, where the power and control cables from the lights were supposed to terminate, were not installed in the positions shown in the CAD models at the time the lights were to be installed as seen in Figure 8. The installation of the internal lighting system involved the first team to travel from offsite to JSC to perform integration, and the HDU team had not yet come up to speed on meeting expectations for visiting teams.
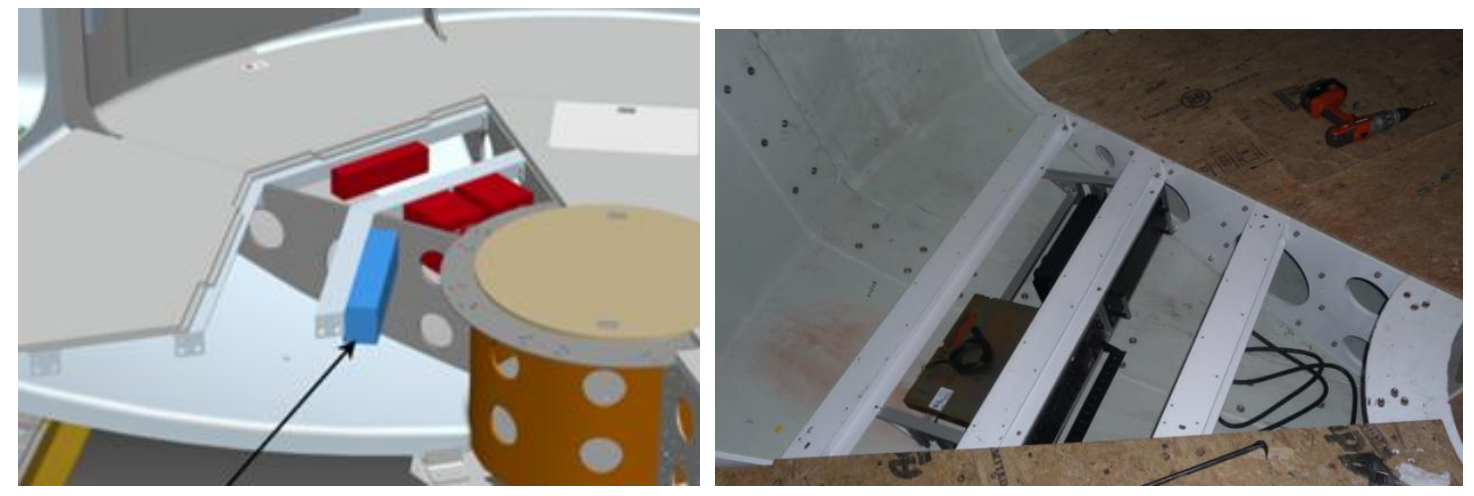

Figure 8 - At left, long red box was proposed terminal box location. At right, the absence of the terminal box at the planned installation timeframe

Although not having the terminal box and controller installed seemed to be a setback, the team actually benefitted because they were able to designate a better location for routing cable into the terminal box. The location originally identified for the controller installation was judged to be very difficult for field maintenance access. Because the potential to have to work on the controller was very high, the team decided the best course of action was to install the controller on the opposite side of the beam selected for installation. Later, that engineering change in the field was relayed to the CAD integrator to update the CAD model with the field implementation. So the lesson learned was though CAD integration is an excellent approach to plan work, there is still significant value in field evaluation.

Additionally, an integrated set of schematics was developed using AutoCAD to both plan the wiring of the systems and to reflect wiring updates made during installation operations. The schematic displayed all the subsystems that required power, control, or both and included wire routing and labeling. Although the team had a good understanding to how and where the cable routing would occur, when installation of the interior lights and respective cabling was being performed, the schematic labeling was not referenced. As a result, different labeling had been created and shrunk wrapped onto the power and control cables for each light. Because the labeling on the schematic and cables were now different, the schematic required an update. Although this didn't create a tremendous amount of extra work, the lesson learned was for future subsystem teams to reference the schematic during integration to ensure the hardware and schematic reflect the same labeling.

The next area to discuss applicable lessons learned is integration and testing. When it came to the physical integration, some of the lessons learned were in the areas of procurement, testing, and scheduled labor for 
integration. Issues in these areas resulted in changes to the overall integration schedule, which led to difficulty meeting deadlines to perform testing at the JSC Rock Yard and Desert RaTS testing.

Due to the number of subsystems contributed by various NASA centers, integration efforts posed a challenge. Despite organized integration efforts using a detailed schedule, many subsystem teams experienced slips in schedule due to such things like long lead times on items. One common problem for many teams was the availability of technician labor and the space in which to do the work because of parallel operations. The tendency for many system teams was to perform their integration late in the schedule, which tied up resources. Space and labor must be taken into account during both integration and testing. On another scheduling note, as integration progressed, last minute procurements became common practice. Late procurements affected labor scheduling problems as well because the late procurements required teams to move dates scheduled for integration, which usually meant labor was already being used to integrate another team.

As the integration time for each system approached, some teams weren't fully prepared for integration because of limited resources or late funding disbursements. As milestones to support the field test activities approached, testing of several subsystems was delayed to the field test phase. As a result, many of the unexpected issues that came up in the field had to be dealt with then instead of in a much more suitable work environment in JSC Building 220 where the initial assembly and integration work occurred. Subsystem teams can increase chances of success by following schedules to the best of their ability to ensure proper procurement, testing, labor scheduling, and integration take place. Additionally, the forward recommendation from the HDU-PEM deployment is to maintain discipline adhering to the original integration plans thus eliminating requirements creep during integration.

Each team needs to hold themselves accountable to meeting procurement, testing and integration schedules because when one team slips and misses procurement, testing, or integration dates, the adjustment in schedule creates a domino effect throughout the project which results in the overall project partially or completely missing important deadlines and milestones.

In the thermal area, one important area of lessons learned was in the selection of the heat pump utilized for the HDU-PEM for field operations. The HDU utilized a standard commercial, residential heat pump for the thermal conditioning of both avionics and crew. The heat pump was mounted on a cradle to facilitate connections and transportation. The thermal system provided air flow into the sub-floor of the HDU where the avionics and power systems were located, and that sub-floor area was used as a plenum where the flow continued from a set of vents into the crew compartment. From there, two sets of return vents cycled air back to the heat pump. Figure 9 depicts the thermal systems air flow scheme for the HDU PEM. The heat pump procured by the HDU team was utilized successfully both at Johnson Space Center Building 220 where all integration activities occurred, and it was also transported about one mile and used outside at the JSC Rock Yard as well. However, after transportation from Houston to Arizona and a three mile trip on dirt roads to the test site, the heat pump condenser manifold did not survive and was fatigued in so many places that the freon leaks could not be repaired. A replacement was borrowed from other Desert RaTS participants to continue operations. And the lesson captured from this episode was to procure a ruggedized unit that was made for transportation and field use.

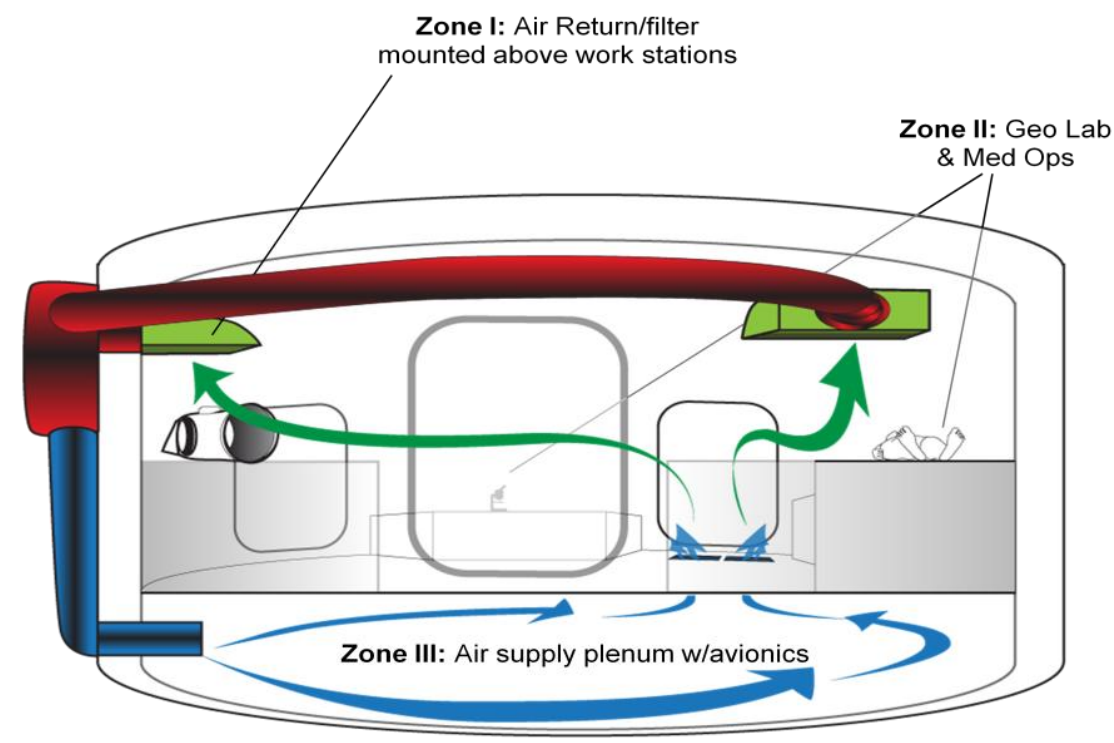


Figure 9 - HDU-PEM Thermal Systems Air Flow

A final lesson learned concerns modularity. After integration of the HDU, the team came away with a conclusion about the modular construction of the HDU. Initially, the steel ribs that were built into the design of the shell to provide a means for bolting each section together and for providing attach points for subsystem integration. Although the ribs served their dual purpose of shell assembly and secondary structure attachment points, the team was limited in their ability to flexibly move workstations or utilities because of the rib protrusions. The lesson learned for future habitats is to strive for a modular configuration where the design of attach points minimally interferes with subsystem integration and cable or utility routing. Another possible solution is to design ribs that have pass through points for cabling and other hardware but to restrain the size of the holes so they don't compromise the structural integrity of the ribs. The orange slice construction of the HDU shell would not be recommended for flight systems design, but this concern with the ribs may be relevant for other analog habitats that are built to be functional at minimal cost.

\section{Conclusion}

The Habitat Demonstration Unit project constructed a habitat shell to act as a test bed for instances of a habitation architecture. For 2010, the HDU acted as a Pressurized Excursion Module of the lunar architecture, and was tested during the 2010 NASA Desert Research and Technology Studies (RaTS) campaign as depicted in CAD in Figure 10 and in photos in Figure 11. Significant challenges to accelerate from a project start to a field deployment in just over a year were addressed with an HDU PEM integration strategy. Efforts were made to define and document standards for system integration, which are valuable not only for the PEM but future versions of the HDU. Additionally, the HDU project utilized a rapid prototyping approach which included the use of computer aided design in the layout of systems, collaboration tools, tailored design review and test processes. And the entire concept of operations from the planning of the manufacturing, shipment, and integration to the field operations were all factored into the design of the HDU to streamline the integration activities allowing the project team to meet the ambitious timeline for deployment of a PEM for Desert RaTS 2010.

An organized effort to evaluate lessons learned from the activities in designing and deploying the HDU PEM have informed and will continue to influence future work for the HDU team,

Looking forward, during fiscal year 2011, the HDU will evolve as a Habitat-Laboratory combination featuring an inflatable loft provided through the X-Hab Challenge. This version of the HDU is called the Deep Space Habitat (DSH). The DSH is an element of a flexible architecture that may enable exploration of Near Earth Objects, and that architecture will be evaluated at Desert RaTS 2011. For additional detail on the HDU Deep Space Habitat configuration, see Ref. 4. For more details on the X-Hab challenge, reference http://www.spacegrant.org/xhab/. The same integration strategies discussed in this paper will be applied during FY11 to enable the utilization of the HDU DSH as a platform for the exploration architecture validation.
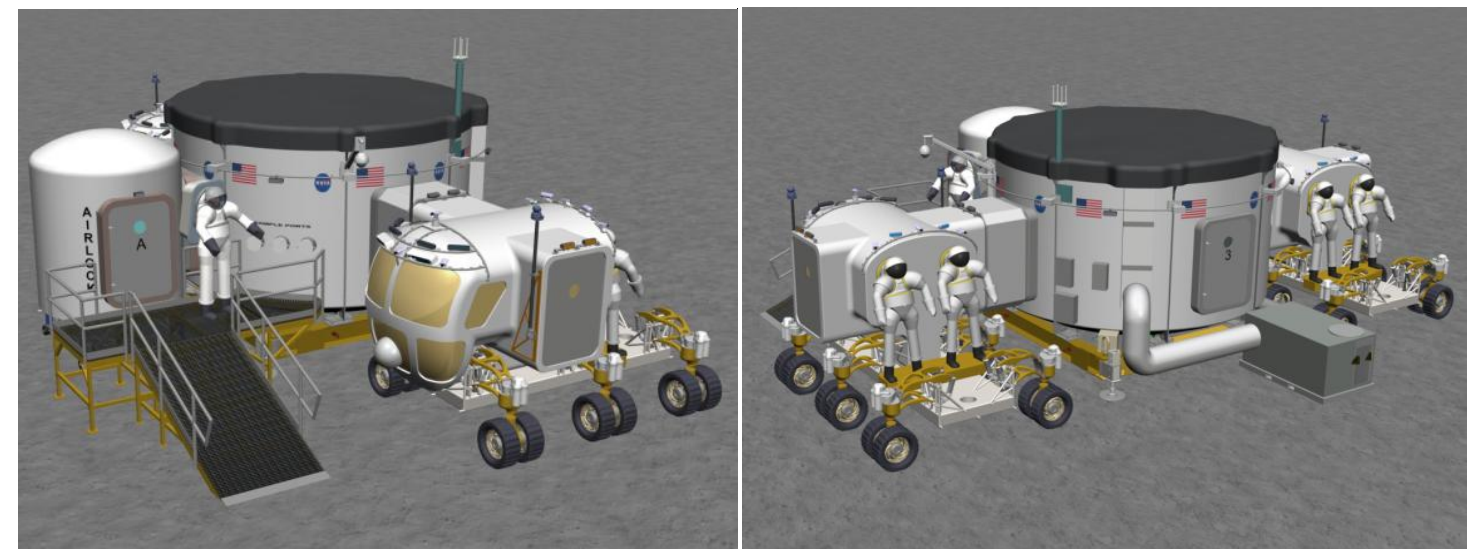

Figure 10 - CAD of Assembled HDU PEM Mounted on Cradle in Configuration for Desert RaTS 2010 


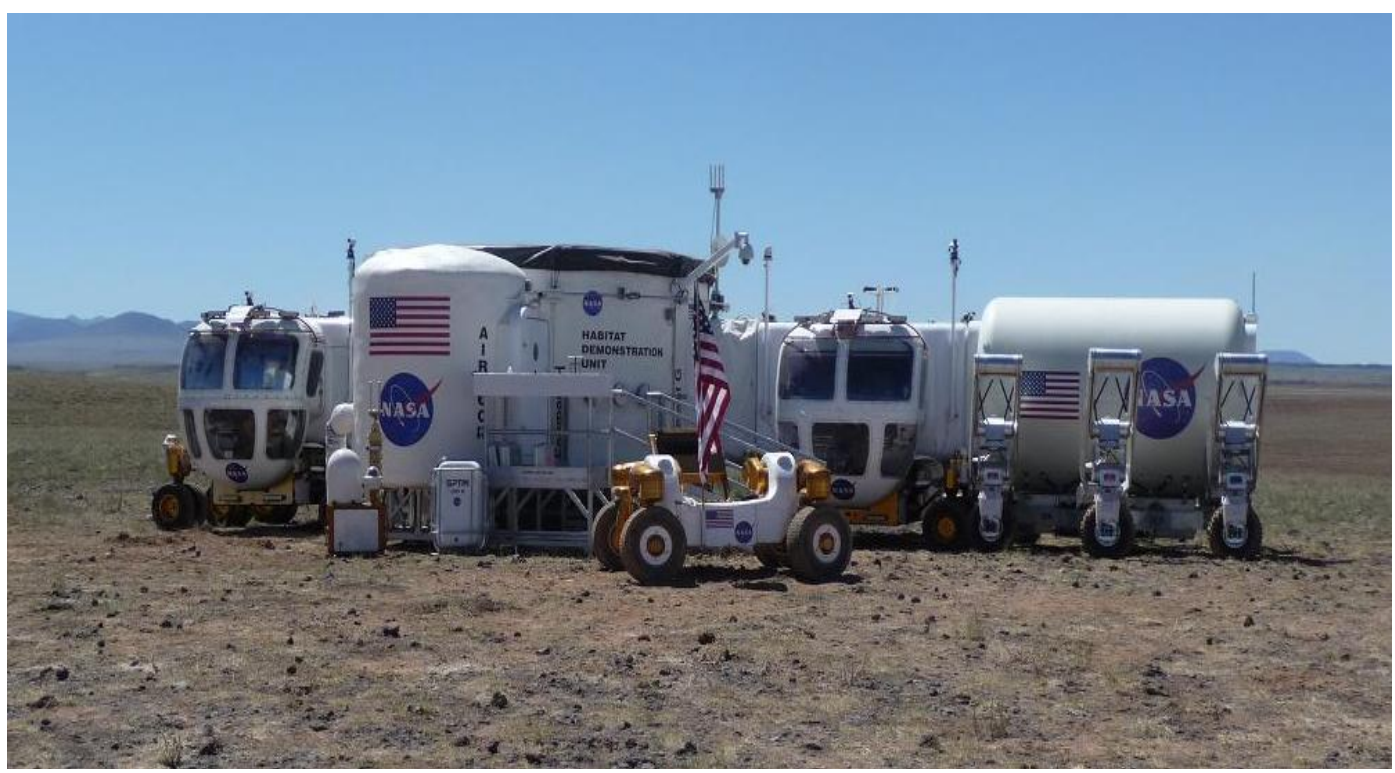

Figure 11 - Space Exploration Vehicle Rovers docked with HDU PEM at Desert RaTS 2010.

\section{References}

1. Craig, D. (2011). "Rapid Prototyping and Analog Testing for Human Space Exploration." NASA Ask Magazine, Issue 42, Spring 2011, pp. 14-18.

2. Howe, A. S., Spexarth, G. R., Toups, L. O., Howard, R. L., Rudisill, M., Dorsey, J. T. "Constellation Architecture TeamLunar Scenario 12.1 Habitation Concept.” Proceedings of ASCE Earth and Space 2010, March 14-17, 2010. ASCE, Reston VA.

3. Tri, T. O., Kennedy, K. J., Gill, T. R., and Howe, A. S. (2011). "The Habitat Demonstration Unit (HDU) Pressurized Excursion Module (PEM) In-Field Demonstration at Desert RATS 2010." Proceedings of the 41st International Conference on Environmental Systems (ICES 2011); July 18-21, 2011. AIAA, Reston, VA.

4. Kennedy, K. J., Gill, T. R., Toups, L. O. Tri, T. O., Smitherman, D. V., and Howe, A. S. (2011). "Habitat Demonstration Unit Project - Deep Space Habitat Overview." Proceedings of the 41st International Conference on Environmental Systems (ICES 2011); July 18-21, 2011. AIAA, Reston, VA. 